\title{
Levetiracetam Ameliorates L-DOPA-Induced Dyskinesia in Hemiparkinsonian Rats Inducing Critical Molecular Changes in the Striatum
}

\author{
Huan Du, ${ }^{1,2}$ Shuke Nie, ${ }^{1}$ Guiqin Chen, ${ }^{1}$ Kai Ma, ${ }^{1}$ Yan Xu, ${ }^{1}$ Zhentao Zhang, \\ Stella M. Papa, ${ }^{4}$ and Xuebing Cao ${ }^{1}$ \\ ${ }^{1}$ Department of Neurology, Union Hospital, Tongji Medical College, Huazhong University of Science and Technology, \\ 1277 Jiefang Avenue, Wuhan 430022, China \\ ${ }^{2}$ Department of Neurology, Sanmenxia Central Hospital, Sanmenxia 472000, China \\ ${ }^{3}$ Department of Neurology, Renmin Hospital, Wuhan University, Wuhan 430060, China \\ ${ }^{4}$ Yerkes National Primate Research Center, Department of Neurology, Emory University School of Medicine, Atlanta, GA 30329, USA
}

Correspondence should be addressed to Yan Xu; xuyanwxf@126.com and Xuebing Cao; caoxuebing@126.com

Received 9 December 2014; Accepted 7 January 2015

Academic Editor: Heinz Reichmann

Copyright (C) 2015 Huan Du et al. This is an open access article distributed under the Creative Commons Attribution License, which permits unrestricted use, distribution, and reproduction in any medium, provided the original work is properly cited.

\begin{abstract}
L-DOPA-induced dyskinesias (LID) remain a major problem of long-term therapy of Parkinson's disease. Levetiracetam, a new antiepileptic drug, has been shown to reduce LID, but the mechanisms underlying its effects are unknown. In this study, we assessed the effect of levetiracetam on key mediators of LID in rats with 6-hydroxydopamine (6-OHDA) lesions. Following chronic administration of L-DOPA ( $12 \mathrm{mg} / \mathrm{kg}$, twice daily for 14 days), rats developed abnormal involuntary movements (AIMs), but coadministration of levetiracetam $(15,30$, and $60 \mathrm{mg} / \mathrm{kg})$ with equivalent L-DOPA dosing significantly reduced AIMs scores in a dose dependent manner. The effects of levetiracetam were associated with changes in striatal expression of $\Delta$ FosB, phosphorylated extracellular signal-regulated kinases 1 and 2 (p-ERK1/2), and phosphorylated cAMP-regulated phosphoprotein of $32 \mathrm{kDa}(\mathrm{p}-$ DARPP-32). These data support that levetiracetam acts at multiple sites in the pathogenetic cascade of LID, and that further understanding of these actions of antiepileptics may contribute to developing new LID therapies.
\end{abstract}

\section{Introduction}

Dopamine replacement therapy with L-3,4-dihydroxyphenylalanine (L-DOPA), still the most effective treatment for Parkinson's disease (PD), is commonly associated with disabling long-term motor complications, including response fluctuations and L-DOPA-induced dyskinesias (LID) [1]. LID are involuntary choreic and dystonic movements that occurred as early as following 3-6 years of L-DOPA treatment in $40 \%$ of PD patients [2]. Furthermore, LID develops in the large majority of patients over the course of chronic exposure to L-DOPA. Available medical treatments for LID produce slight benefits, and in many cases the management of LID ultimately relies on DBS surgery.

Although the pathogenesis of LID remains unclear, it is believed that both disease progression and nonphysiologic pulsatile dopamine stimulus contribute to the associated changes in various signaling pathways and basal ganglia circuits $[3,4]$. Physiologic and molecular studies have shown hypersensitivity of $D_{1}$ receptor-mediated responses in the direct striatal output pathway leading to imbalance of striatal outputs [5]. Moreover, studies of advanced Parkinsonian monkeys have shown abnormal reversal of dopamine responses in direct and indirect output neurons indicating imbalanced discharges across neurons within each pathway [6]. In the direct pathway, increased activation of cAMPdependent signaling and PKA through $\mathrm{D}_{1}$ receptors leads to increased phosphorylation of various downstream proteins, such as ERK1/2 (extracellular signal-regulated kinases 1 and 2) and DARPP-32 (dopamine- and cAMP-regulated phosphoprotein of $32 \mathrm{kDa}$ ) [7, 8]. In addition, transcription factors expressed with chronic events such as $\Delta \mathrm{FosB}$ 
(a truncated splice variant of FosB) are overexpressed in the striatum of rodents and primates with dyskinesias $[9,10]$. Pharmacological or genetic interventions to reduce the levels of these proteins can attenuate LID, as shown in DARPP32-deficient mice or after inhibition of PKA in rats [11, 12]. Furthermore, the transgenic overexpression of $\Delta$ FosB reproduces AIMs in hemiparkinsonian rats without chronic exposure to L-DOPA [13].

Levetiracetam ((S)-a-ethyl-2-oxo-pyrrolidine acetamide, LEV) is an antiepileptic drug that reduces LID in the macaque MPTP model of PD [14]. The action mechanism of LEV seems to be nonconventional for its class because unlike other antiepileptic drugs LEV may predominantly bind to the synaptic vesicular protein SV2A. Additionally, coadministration of L-DOPA and LEV following one-week drug holiday in primates demonstrated significant reduction of LID pointing to effects on L-DOPA priming for LID [15]. However, levetiracetam action on LID remains elusive. Here, we tested levetiracetam effects on those molecules known to be involved in LID mechanisms in rats with unilateral 6OHDA lesions of the striatonigral pathway. Levetiracetam had significant regulatory effects on $\Delta$ FosB, p-ERK1/2, and p-DARPP-32 expression, which correlated with reduction of abnormal involuntary movements (AIMs) demonstrating the specific effects of this antiepileptic drug on dyskinesia mechanisms.

\section{Materials and Methods}

2.1. Unilateral 6-OHDA-Lesioned Model. Male SpragueDawley (SD) rats were bought from the Experimental Animal Center of Tongji Medical College at Huazhong University of Science and Technology (200-250 g), housed in controlled environment ( $12 \mathrm{hrs} \mathrm{light/dark} \mathrm{cycle,} 22 \pm 2^{\circ} \mathrm{C}, 55 \pm 5 \%$ relative humidity) and allowed free access to food and water. All of the experimental protocols were approved by the Institutional Animal Care and Use Committee of Huazhong University of Science and Technology (HUST). Rats were anesthetized with $10 \%$ chloral hydrate $(0.5 \mathrm{~mL} / 100$ g, i.p. $)$ and injected unilaterally in the medial forebrain bundle with $4 \mu \mathrm{L}$ of $6-\mathrm{OHDA}(2 \mathrm{mg} / \mathrm{mL}$, dissolved in saline/ascorbic acid, Sigma-Aldrich, St. Louis, MO) following stereotaxic coordinates (AP + 4.0, L +1.3, DV-8.4 mm from the interaural line) at a rate of $0.5 \mu \mathrm{L} / \mathrm{min}$ using a micropump [16].

Three weeks after surgery, rats were injected subcutaneously with a subthreshold dose of apomorphine $(0.05 \mathrm{mg} / \mathrm{kg})$ to test for a rotational response indicative of supersensitivity developed with full denervation. Rats exhibiting an average of $\geqslant 7$ turns/min were selected for the study and randomly divided in four groups of 10 rats each: (A) control group, (B) $15 \mathrm{mg}$ levetiracetam group, (C) $30 \mathrm{mg}$ levetiracetam group, and (D) $60 \mathrm{mg}$ levetiracetam group (see Figure 1).

2.2. Drug Treatment. Rats were pretreated by gavage with levetiracetam $(15,30$, or $60 \mathrm{mg} / \mathrm{kg})$ as previously described [14] and two hours later injected intraperitoneally with L-DOPA methyl ester $(12 \mathrm{mg} / \mathrm{kg})$ plus benserazide- $\mathrm{HCl}(3 \mathrm{mg} / \mathrm{kg})$ dissolved in saline (Sigma-Aldrich, Madrid, Spain) twice daily
(9 AM and 5 PM) for 14 days [17]. The control group received saline before L-DOPA treatment.

2.3. Behavioral Measurements. Animals were assessed on days $1,3,5,7,9,11$, and 14 following a challenge of L-DOPA methyl ester $(12 \mathrm{mg} / \mathrm{kg})$ plus benserazide- $\mathrm{HCl}(3 \mathrm{mg} / \mathrm{kg})$ (i.p.). Rats were assessed individually at 30, 60, 90, and 120 min after the L-DOPA injection using an adaptation of the AIMs Scale [18]. The four subscales (oral dyskinesia, limb dyskinesia, axial dystonia, and contraversive rotation) were used to score: (1) orolingual dyskinesia, stereotyped jaw movements, and tongue protrusion, (2) forelimb dyskinesia, repetitive rhythmic jerks, and contralateral to the lesion side, (3) axial dystonia, contralateral twisted posturing of the trunk, neck, and head, and (4) rotational behavior. Animals were scored every 30 minutes for 2 hours after treatment. The frequency and intensity of AIMs were evaluated using the following rating: 0 , absent; 1 , occasional; 2, frequent; 3 , continuous but interrupted by external stimuli; and 4, continuous and not interrupted by external stimuli. Rotational tests were carried out in a cylinder and only completed turns $\left(360^{\circ}\right)$ were counted. All behavioral tests were done in a blinded fashion. Two hours after the last L-DOPA injection, the animals were sacrificed for immunocytochemistry and western blotting analyses.

2.4. Tissue Preparation and Immunocytochemistry. After completing the above behavioral tests, rats $(n=4)$ were killed under anesthesia and immediately perfused transcardially with $4 \%$ paraformaldehyde (dissolved in PBS). The brains were fixed in $4 \%$ buffered formalin and embedded in paraffin for sectioning. Coronal sections $(5 \mu \mathrm{m})$ were cut and stained with hematoxylin/eosin (H\&E) for histopathological evaluation. Fixed brain sections were deparaffinized and hydrated. After antigen-retrieval in boiling $10 \mathrm{mM}$ sodium citrate for $20 \mathrm{~min}$, sections were incubated in rabbit polyclonal antiFosB (1:2000, Santa Cruz, CA, USA) overnight at $4^{\circ} \mathrm{C}$ and then incubated in the avidin-biotin peroxidase complex using the appropriate rabbit $\mathrm{ABC}$ Vectastain kit (Vector Laboratories, USA). Immunoreactivity was observed using 3, 3-diaminobenzidine (DAB, Sigma, USA) as the chromagen. Positive immunoreactive cells were counted under the microscope in five randomly chosen high-magnification fields $(\times 200)$ in the striatal region and analyzed using the software Imagine J by an independent experimenter blind to the sections.

2.5. Western Blotting. After anesthesia with $10 \%$ chloral hydrate of the remaining rats, tissue samples harvested from the bilateral dorsal striatum were extracted with lysis buffer containing protease inhibitors, homogenized, and centrifuged. Supernatants yielding $30 \mu \mathrm{g}$ protein were subjected to $10 \%$ SDS-polyacrylamide gels and electrophoretically transferred to polyvinylidene difluoride (PVDF) membranes. Membranes were incubated with specific primary antibodies: polyclonal goat-anti phosphor-DARPP-32 at 


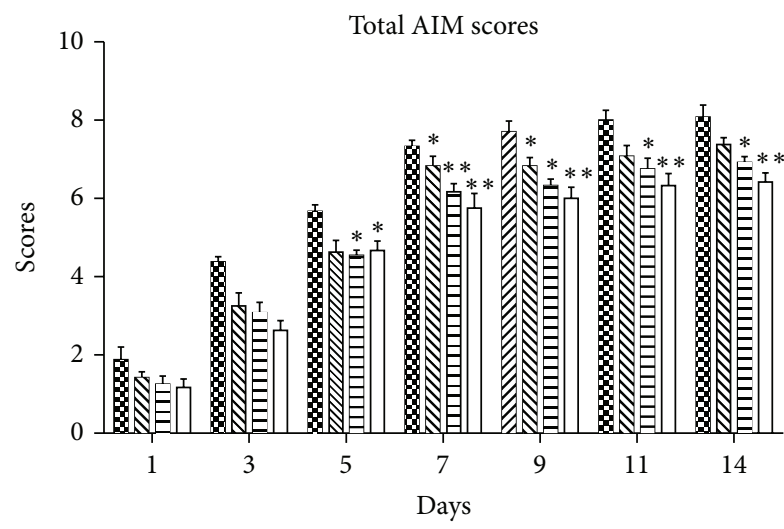

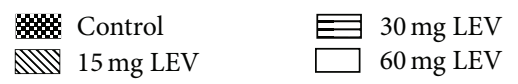

(a)

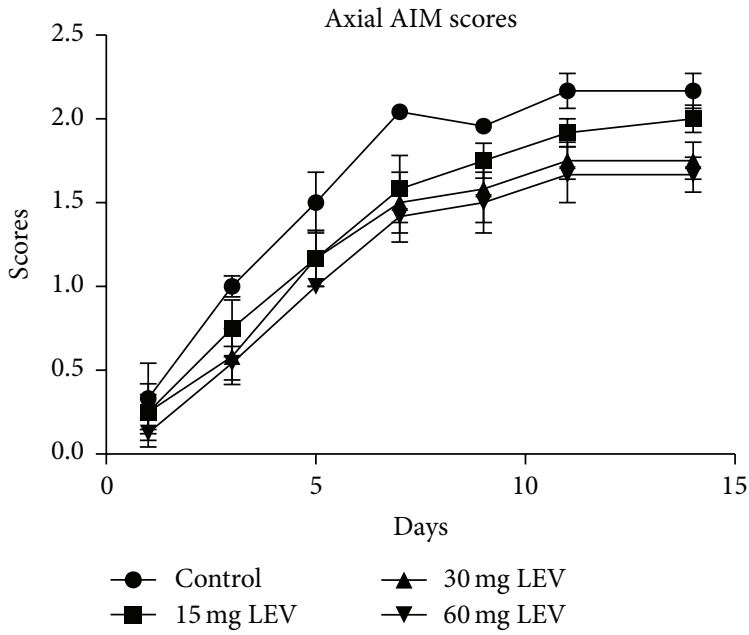

(c)

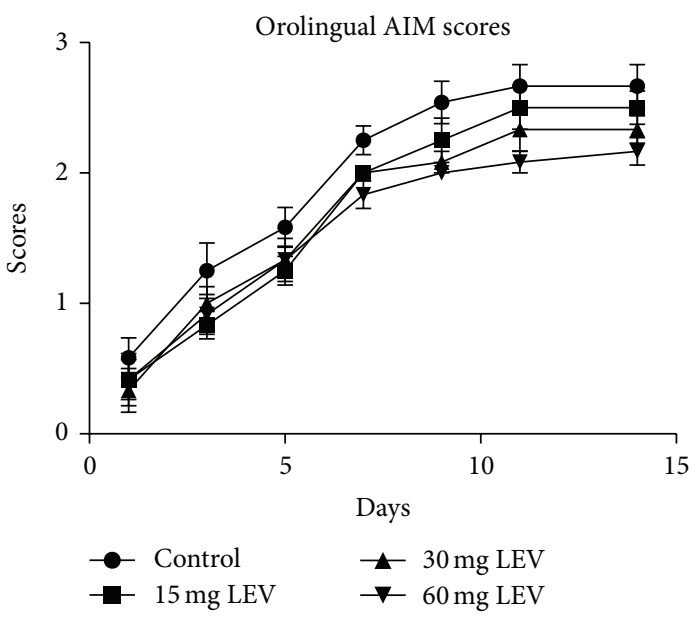

(b)

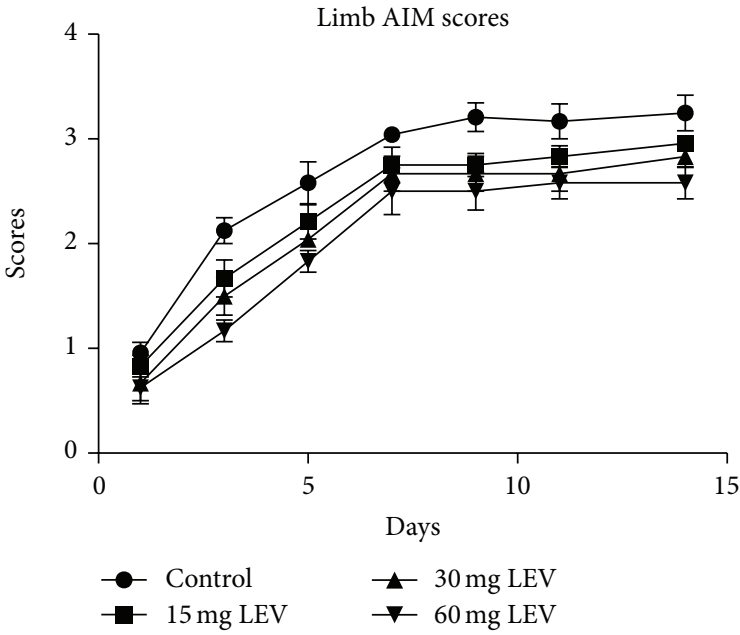

(d)

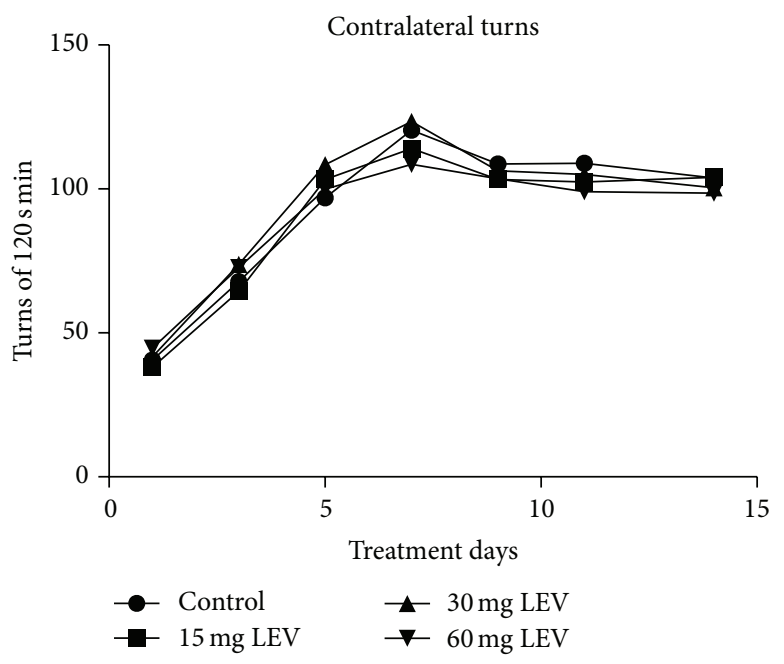

(e)

FIGURE 1: Effects of levetiracetam on abnormal involuntary movements (AIMs) induced by L-DOPA in hemiparkinsonian rats. Animals were assessed on days 1, 3, 5, 7, 9, 11, and 14 following L-DOPA methyl ester (12 mg/kg) plus benserazide- $\mathrm{HCl}(3 \mathrm{mg} / \mathrm{kg})$ (i.p.). Two hours prior to each L-DOPA injection, animals received levetiracetam 0 (control group, vehicle injection), 15, 30, or $60 \mathrm{mg} / \mathrm{kg}$ by gavage. Total AIMs scores (a) include orofacial (b), axial (c), and limb (d) AIMs scores. Rotational behavior (contralateral turns, (e)) shows that levetiracetam induced no changes in the antiparkinsonian action of L-Dopa. Values are mean \pm SEM. ${ }^{*} P<0.05$ and ${ }^{* *} P<0.01$ versus control group $(n=10$ per group; LEV: levetiracetam). 
threonine34 (1:100, Santa Cruz), polyclonal rabbit antiDARPP-32 (1:200, Santa Cruz), polyclonal rabbit antiFosB (1:200, Santa Cruz), polyclonal rabbit anti-ERK1/2 phosphorylated at threonine202/tyrosine204 of ERK1, and threonine185/tyrosine187 of ERK2 (1:1000, Cell Signaling Technology), followed by incubation with horseradish peroxidase-conjugated secondary antibody (ICN Pharmaceuticals). Proteins were visualized with a Super Signal West Pico chemiluminescence kit (Thermo Scientific, USA). $\beta$ actin (1:500, Santa Cruz) was used to normalize protein expression.

2.6. Statistical Analysis. Statistical analysis was performed using SPSS 16.0 software. For AIMs, comparison between groups was made using nonparametric test. For immunocytochemistry and western blotting analysis, Student's $t$ tests were used when only two groups were compared. The differences between groups (more than two groups) were made by two-way analysis of variance (ANOVA) followed by post hoc comparison with Bonferroni test depending on the data. Significance level was set at $P<0.05$. Data are represented as mean \pm SEM.

\section{Results}

3.1. Behavioral Effects (Levetiracetam Reversed AIMs in a Dose-Dependent Manner). L-DOPA treatment induced AIMs that are dyskinetic-like movements in hemiparkinsonian rats, as expected in the unilateral 6-OHDA-lesion model. The chronic administration of L-DOPA $12 \mathrm{mg} / \mathrm{kg}$ twice daily induced gradual development of AIMs over the course of the treatment period. The incidence and intensity of AIMs were maximal from 30 to $60 \mathrm{~min}$ after L-DOPA injection. AIMs scores decreased gradually to reach baseline after 2 to 3 hours from injection. The frequency and severity of AIMs increased significantly during the first 5 to 12 days of L-DOPA treatment. After that time, their intensity remained stable (Figure 1(a)).

By the 5th day of treatment, all levetiracetam doses significantly reduced the total AIMs and forelimb scores compared to the control group, and this effect was maintained until the end of the L-dopa response (Figures 1(a) and 1(b)). Levetiracetam reduced the severity of AIMs particularly in the forelimbs. The effects of levetiracetam 30 and $60 \mathrm{mg}$ on forelimb AIMs were significant in the 3rd day of treatment with score reduction from $2.13 \pm 0.13$ to $1.5 \pm 0.18$ and $1.17 \pm 0.11$, respectively $(n=10$ group, $P<0.05$, Figure 1(b)). Levetiracetam also reduced orolingual dyskinesia and axial dystonia since the 7th day of treatment, but only at the $60 \mathrm{mg}$ dose (Figures 1(c) and 1(d)). The total AIMs scores reduced from $8.08 \pm 0.74$ to $6.92 \pm 0.37$ in the $30 \mathrm{mg}$ levetiracetam group $(P<0.05, n=10$ /group, Figure 1(a)) and to $6.42 \pm 0.58$ in the $60 \mathrm{mg}$ levetiracetam group $(n=$ $10 /$ group, $P<0.01)$. The lowest dose of levetiracetam (15 mg) had a smaller effect on AIMs during the first 10 days of treatment and became nonsignificant after the 11th day. These data indicate a dose-dependent effect of levetiracetam on AIMs.
Levetiracetam did not interfere with the antiparkinsonian action of L-DOPA. Contralateral rotation, a measurement of such an L-Dopa action, increased during the first week of daily L-DOPA treatment and stabilized in the second week with no differences across the control and levetiracetam treatment groups (Figure 1(e)). These behavioral data are aligned with previous reports showing no differences in Parkinsonian motor disability or "on-time" effect [14]. These results indicate that levetiracetam effect on AIMs is not related to diminishment in the beneficial antiparkinsonian action of L-DOPA.

3.2. Molecular Effects (Levetiracetam Decreased the Expression of $\triangle F O S B$ and the Phosphorylation of DARPP-32 and $E R K 1 / 2$ in the Striatum). FosB/ $\triangle$ FosB immunoreactive neurons increased in the dorsolateral part of the striatum on the lesion side with the used antibody that recognizes all members of the FosB family. All doses of levetiracetam decreased the number of FosB/ $\Delta$ FosB positive cells (from $88.7 \pm 1.7 /$ section in the control group to $65.7 \pm 0.87,42.3 \pm$ 1.88 , and $25.7 \pm 1.2 /$ section in the 15,30 , and $60 \mathrm{mg}$ groups, resp.; Figure 2). These results indicate dose-dependent effects of levetiracetam on FosB/ $\Delta$ FosB expression.

To determine the selective effects of levetiracetam on $\Delta$ FosB levels, striatal tissue was processed for immunoblotting as well. The striatal expression of $\Delta F o s B$, the truncated form of FosB, was exaggeratedly higher in the lesioned side compared to the intact side of the striatum after chronic L-DOPA treatment $(P<0.01$, Figure 3(a)). All doses of levetiracetam reduced striatal $\triangle$ FosB levels from $1.02 \pm 0.02$ in the control group to $0.97 \pm 0.02(P<0.05), 0.83 \pm 0.02$ $(P<0.05)$, and $0.64 \pm 0.01(P<0.01)$ in the 15,30 , and $60 \mathrm{mg}$ levetiracetam treatment group, respectively.

The striatal expression of $\mathrm{p}$-ERK1/2 also increased in the lesioned side compared to the intact side of the striatum after chronic L-DOPA treatment $(P<0.01$, Figure 3(b)). Levetiracetam 30 and $60 \mathrm{mg}$ reduced the striatal expression of p-ERK1/2 (from $0.94 \pm 0.01$ to $0.89 \pm 0.01$ and $0.84 \pm 0.003$, resp., $P<0.05)$.

DARPP-32 phosphorylation at threonine34 increased in the lesioned side compared to the intact side of the striatum $(P<0.01$, Figure 4(a)). Levetiracetam $60 \mathrm{mg}$ significantly reduced the striatal expression of p-DARPP-32 (0.56 \pm 0.01 versus $0.47 \pm 0.01, P<0.01)$. Lower doses of levetiracetam (15 and $30 \mathrm{mg}$ ) had little effect on the phosphorylation of DRAPP-32. Levetiracetam had no effect on total DARPP-32 levels, which were similar between the lesioned and intact side of the striatum $(P>0.05$, Figure 4(b)). Altogether, these data indicate that levetiracetam regulates the striatal expression of molecules involved in the mechanisms of dyskinesias, that is, $\triangle$ FosB, p-DARPP-32, and p-ERK1/2.

\section{Discussion}

In the present study, we found that levetiracetam reduced the development of AIMs, the rodent equivalent of LID, in hemiparkinsonian rats following chronic exposure to LDOPA, and these results in rodents are in agreement with the previous study in MPTP-lesioned macaques showing 

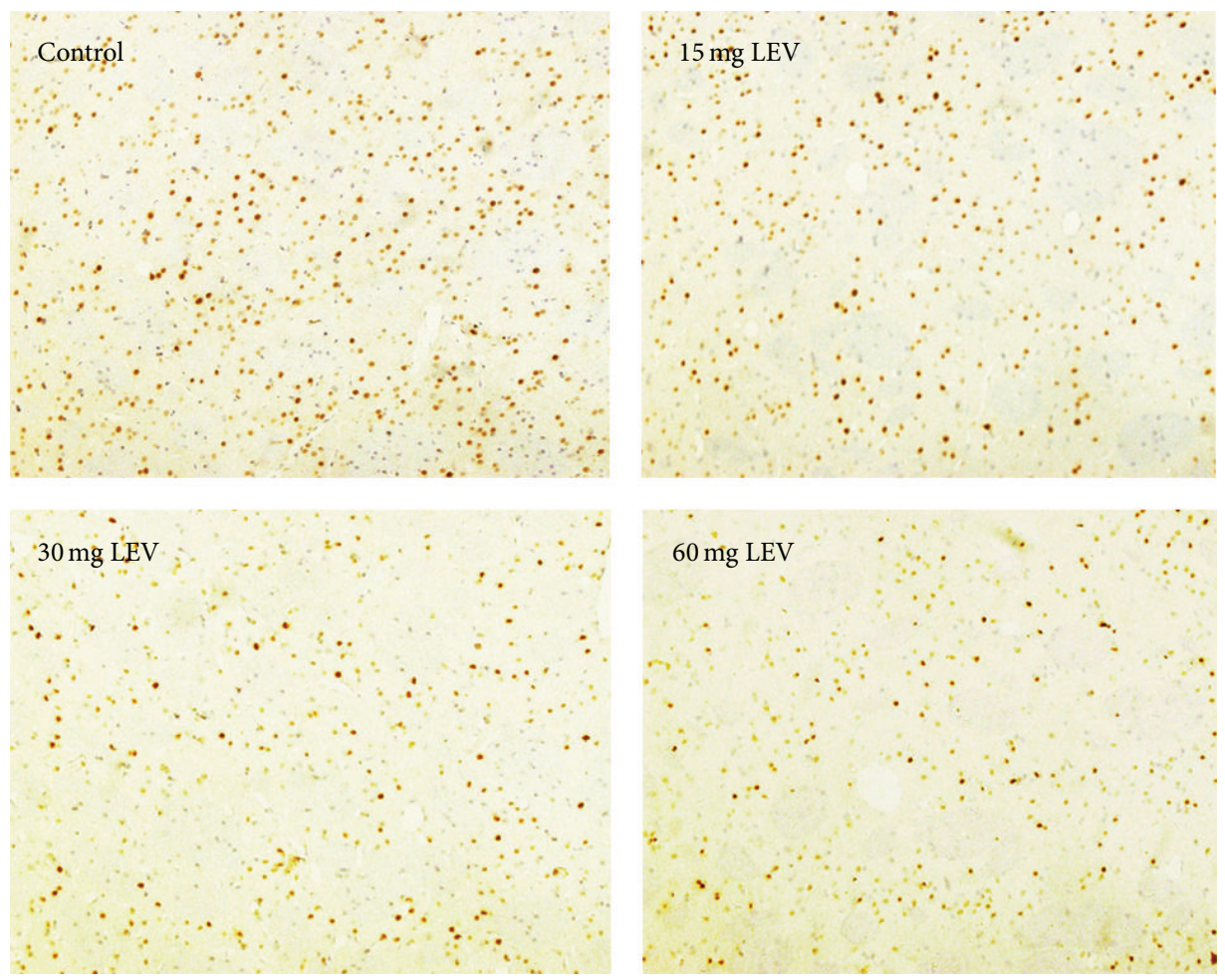

(a)

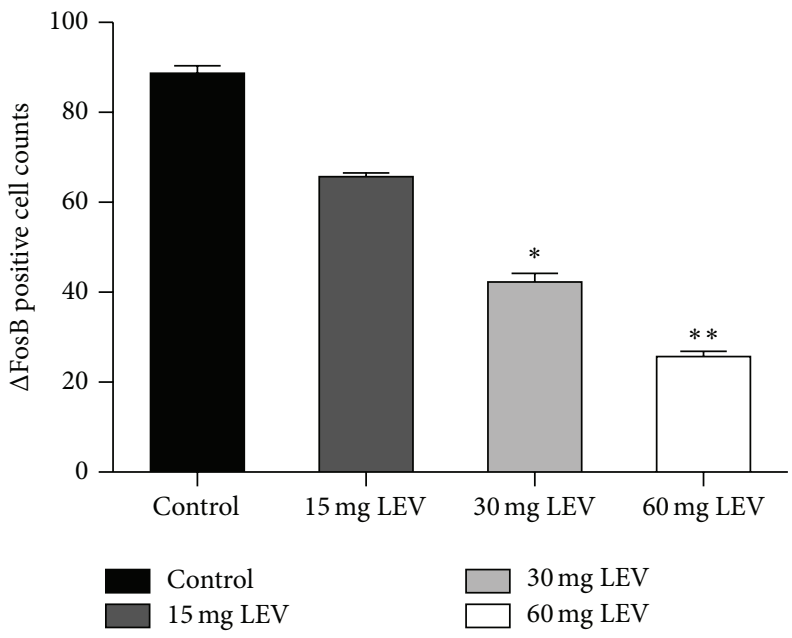

(b)

Figure 2: Levetiracetam-induced changes in FosB/ $\Delta$ FosB immunostaining of the dorsolateral portion of the striatum. (a) Images of striatal slices from the lesion side of an animal from each treatment group (control, 15, 30, and $60 \mathrm{mg} / \mathrm{kg}$ levetiracetam denoted as LEV; $\times 200$ ). (b) Immunoreactive cell count in each treatment group. Values are mean \pm SEM. ${ }^{*} P<0.05$ and ${ }^{* *} P<0.01$ versus control group $(n=4$ per group).

effects on established LID as well as L-DOPA priming for LID $[14,15]$. The development of AIMs was associated with increased $\triangle$ FosB expression and $\mathrm{p}$-ERK1/2 and DARPP-32 phosphorylation in the striatum ipsilateral to the lesion. The most striking change was in $\triangle$ FosB levels that increased 2-3 fold in the denervated side compared to the intact side. Our data showed that the molecular changes associated with LID were reversed by levetiracetam, and it should be noted that the effects of LVT appear limited.
The exact pathogenetic mechanisms underlying LID are still unclear; however, the evidence indicates that exogenous L-DOPA given with a pulsatile regimen is converted to dopamine irregularly in the remaining dopamine terminals and neighbor cells leading to nonphysiologic dopamine release in the striatum and thereby fluctuating stimulation on postsynaptic dopamine receptors [19-21]. This abnormal stimulation may cause imbalance of dopamine responses in striatal output neurons [3]. Studies in rodent and 

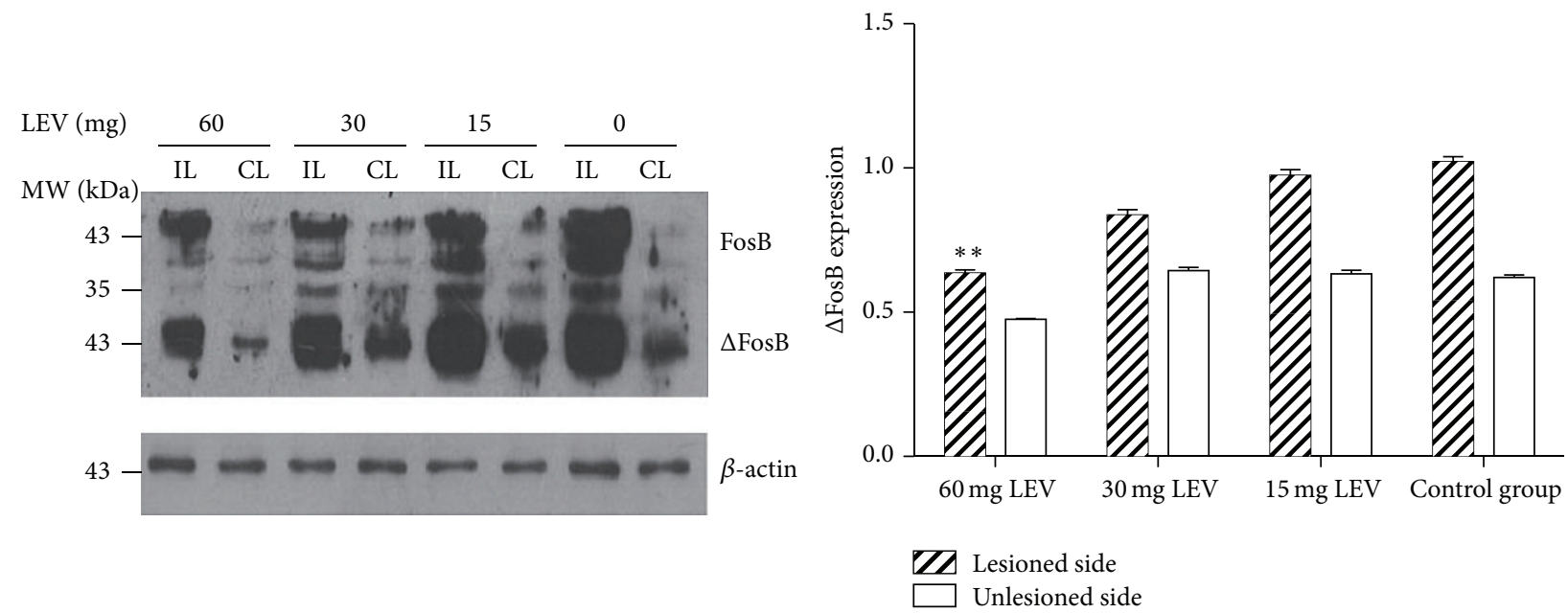

(a)
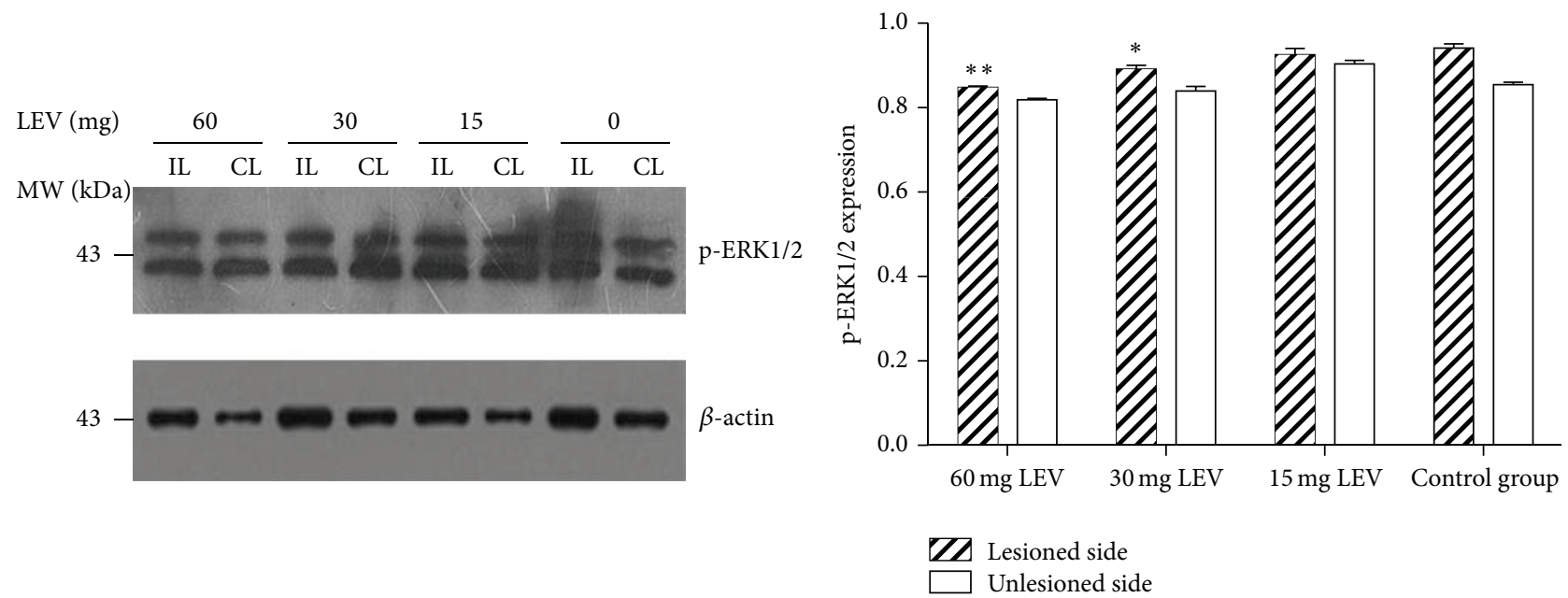

(b)

FIGURE 3: Levetiracetam-induced expression changes of the $\Delta \mathrm{FosB}$ and phosphorylation of ERK1/2 in the dorsolateral portion of the striatum. Images display immunoblots from striatal tissue (IL: ipsilateral side; CL: contralateral side) from one animal in each levetiracetam treatment group, and immunoblots correspond to FosB, $\Delta \mathrm{FosB}$, and $\beta$-actin as internal control. Levetiracetam $60 \mathrm{mg} / \mathrm{kg}$ significantly reduced the striatal $\triangle$ FosB expression (a). ${ }^{\#} P<0.05$ versus same side of control group ( $n=6$ per group; LEV: levetiracetam). Images display immunoblots from striatal tissue (IL: ipsilateral side; CL: contralateral side) from one animal in each levetiracetam treatment group, and immunoblots correspond to $\mathrm{p}$-ERK1/2 and $\beta$-actin as internal control. Levetiracetam 30 and $60 \mathrm{mg} / \mathrm{kg}$ significantly reduced the striatal p-ERK1/2 expression (b). ${ }^{*} P<0.05$ and ${ }^{* *} P<0.01$ versus same side of the control group ( $n=6$ per group; LEV: levetiracetam).

primate models have shown that dopamine depletion followed by the subsequent replacement therapy is associated with hypersensitive responsiveness of striatal neurons expressing the dopamine $\mathrm{D}_{1}$ receptors and projecting into the direct output pathway $[6,22] . \mathrm{D}_{1}$ receptor activation increases cyclic adenosine $3^{\prime}, 5^{\prime}$-monophosphate (cAMP), which in turn activates cAMP-dependent protein kinase A (PKA). This enzyme is critical for the phosphorylation of various proteins downstream in the signaling cascade. One of these proteins is DARPP32, its phosphorylation at the threonine- 34 residue is induced by L-DOPA through $\mathrm{D}_{1}$ receptor-PKA-mediated mechanisms. The striatal levels of phospho[Thr34]-DARPP-32 were found to correlate with the severity of dyskinesia in rodents $[22,23]$, and deletion of
DARPP-32 gene significantly reduced AIMs. In addition, DARPP-32, through 2 mitogen-activated protein kinases (MAPKs), ERK1 and ERK2, participates in the regulation of gene expression in the striatum of dyskinetic animals $[7,8]$. Following chronic L-DOPA administration in rats and monkeys, p-ERK1/2 is elevated in striatal medium spiny neurons directly correlating with LID severity, and LID can be attenuated by reducing striatal p-ERK1/2 levels [24]. Other important molecule participating in the mechanisms of dyskiniesias is $\Delta$ FosB, a truncated splice variant of the immediate early gene FosB [9]. Striatal $\Delta$ FosB expression increases significantly following dopamine denervation, and it elevates to even higher levels with chronic L-DOPA treatment in rats and macaques correlating with dyskinesia 

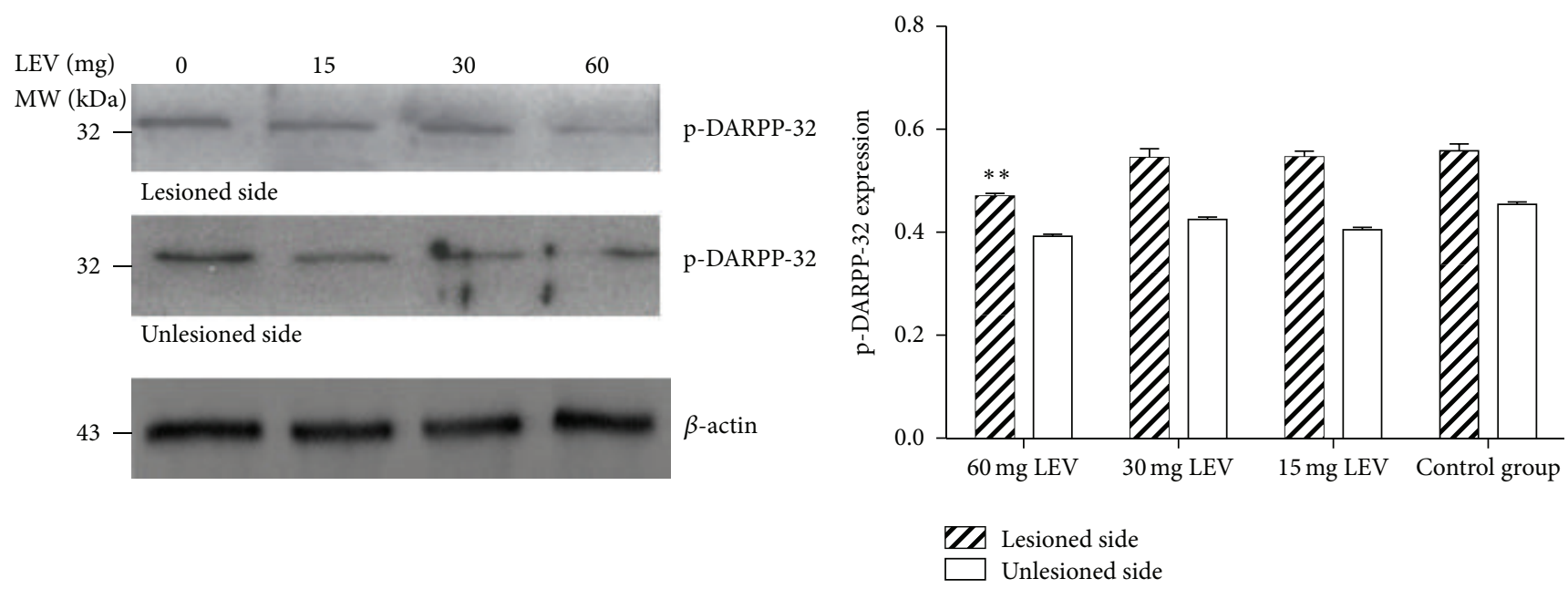

(a)
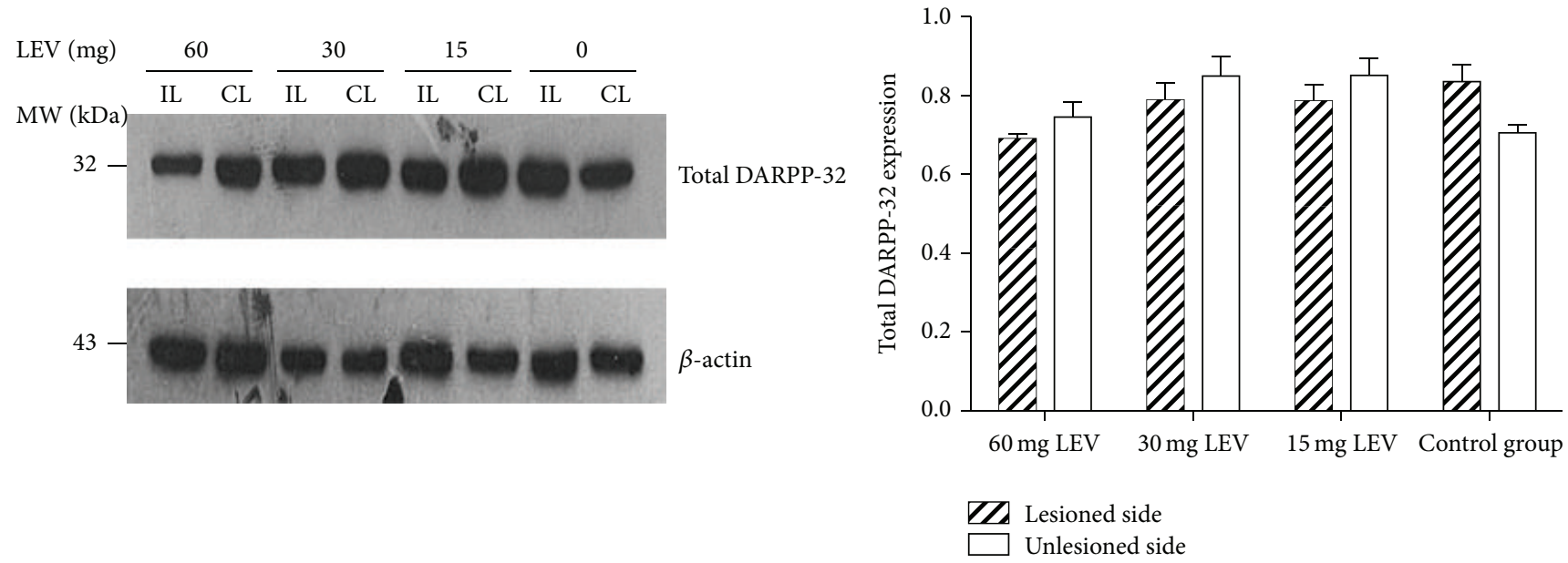

(b)

FIGURE 4: Levetiracetam-induced changes in the phosphorylation of DARPP-32 in the dorsolateral portion of the striatum. (a) Images display immunoblots from striatal tissue (lesioned and unlesioned side) from one animal in each levetiracetam treatment group. Immunoblots correspond to p-DARPP-32 and $\beta$-actin as internal control. The graph compares p-DARPP-32 expression across treatment groups. Levetiracetam $60 \mathrm{mg} / \mathrm{kg}$ significantly reduced the striatal p-DARPP-32 expression. ${ }^{* *} P<0.01$ versus same side of the control group. (b) Images display immunoblots from striatal tissue (IL: ipsilateral side; CL: contralateral side) from one animal in each levetiracetam treatment group. Immunoblots correspond to total DARPP-32 and $\beta$-actin as internal control. The graph compares total DARPP-32 expression across treatment groups. Levetiracetam had nonsignificant effects on the striatal total DARPP-32 expression ( $n=6$ per group; LEV: levetiracetam).

severity $[10,13,25]$. Furthermore, $\Delta$ FosB overexpression has been observed in postmortem striatal studies of Parkinsonian patients chronically treated with L-DOPA [26].

Our data showed that levetiracetam had specific effects on striatal $\Delta$ FosB, p-DARPP-32, and p-ERK1/2, and these molecular effects were parallel to the LID changes. Also both the molecular and behavioral effects were dose-dependent. Levetiracetam is a new type of antiepileptic drug, which binds to ion channels and specifically to the presynaptic vesicle protein SV2A participating in neurotransmitter release. It has been proved efficient as adjunctive therapy in patients with partial seizures [27]. In mechanistic studies, it was found that this antiepileptic drug can activate the ROMK1 channel through PKA phosphorylation as well [28]. An effect on PKA phosphorylation may also be involved in the antidyskinetic actions of levetiracetam. It is plausible that levetiracetam acts at multiple sites in the molecular pathways associated with LID development [29]. Of note, the most prominent effect of levetiracetam was the reduction of $\Delta \mathrm{FosB}$ expression, which cannot be explained by any of its known actions on vesicular protein or ion channels. Therefore, the exact mechanism(s) underlying the antiepileptic effects of levetiracetam remains uncertain. The present study revealed that levetiracetam induces striatal molecular changes that are tightly linked to the occurrence of LID and, therefore, levetiracetam can specifically elicit antidyskinetic effects.

Several open-label clinical trials have confirmed efficacy of levetiracetam for the management of levodopa-induced dyskinesias in PD $[30,31]$. In addition, in a randomized, double-blind, placebo-controlled, pilot study in patients with 
moderate-to-severe LID, levetiracetam provided significant improvement of dyskinesias [32]. As in these limited clinical trials, the present study and other tests in animal models showed that levetiracetam has antidyskinetic effects without affecting the antiparkinsonian action of L-DOPA [33]. This pharmacological profile together with its specific effects on molecular mediators of LID supports the therapeutic potential of this drug and others in the same class for PD. Furthermore, the present data support further studies into the mechanisms of action of levetiracetam, particularly to investigate its role on modulation of striatal PKA signaling, corticostriatal plasticity and the abnormal medium spiny neuron activity in association with dyskinesias $[3,6,34]$.

\section{Conclusion}

In summary, we have demonstrated that Levetiracetam can attenuate AIMs dose-dependently in 6-hydroxydopamine lesioned rats. Levetiracetam reverses the striatal $\Delta$ FosB overexpression and reduces the striatal levels of phosphorylated ERK1/2 and DARPP-32, associated with the improvement of LID. This study supports that levetiracetam acts at multiple sites in the pathogenetic cascade of LID and that further understanding of these actions of antiepileptics may contribute to develop new LID therapies.

\section{Conflict of Interests}

None of the authors has any type of conflict of interests with this report. Dr. Papa has received research support from NIH, Michael J. Fox Foundation, Pfizer, Inc., EnVivo Pharmaceuticals, Inc., Forum Pharmaceuticals, Inc., GeneGraft, LTD, and Key Neurosciences. She is a consultant for Teva Neuroscience.

\section{Authors' Contribution}

Huan Du and Shuke Nie contributed equally to this work.

\section{Acknowledgments}

This research was supported by the National Natural Science Foundation of China (NSFC Project No. 81171193 and No. 370700881). SMP is supported by NIH grants NS045962, NS073994, NCRR RR000165 and ORIP/OD OD011132.

\section{References}

[1] J. A. Obeso, C. W. Olanow, and J. G. Nutt, "Levodopa motor complications in Parkinson's disease," Trends in Neurosciences, vol. 23, no. 10, pp. S2-S7, 2000.

[2] E. Bezard, J. M. Brotchie, and C. E. Gross, "Pathophysiology of levodopa-induced dyskinesia: potential for new therapies," Nature Reviews Neuroscience, vol. 2, no. 8, pp. 577-588, 2001.

[3] P. Jenner, "Molecular mechanisms of L-DOPA-induced dyskinesia," Nature Reviews Neuroscience, vol. 9, no. 9, pp. 665-677, 2008.

[4] P. Calabresi, M. di Filippo, V. Ghiglieri, and B. Picconi, "Molecular mechanism underlying levodopa-induced dyskinesia," Movement Disorders, vol. 23, supplement 3, pp. S570-S579, 2008.
[5] C. R. Gerfen, "D1 dopamine receptor supersensitivity in the dopamine-depleted striatum animal model of Parkinson's disease," The Neuroscientist, vol. 9, no. 6, pp. 455-462, 2003.

[6] L. Liang, M. R. DeLong, and S. M. Papa, "Inversion of dopamine responses in striatal medium spiny neurons and involuntary movements," The Journal of Neuroscience, vol. 28, no. 30, pp. 7537-7547, 2008.

[7] C. R. Gerfen, S. Miyachi, R. Paletzki, and P. Brown, "D1 dopamine receptor supersensitivity in the dopamine-depleted striatum results from a switch in the regulation of ERK1/2/MAP kinase," Journal of Neuroscience, vol. 22, no. 12, pp. 5042-5054, 2002.

[8] J. E. Westin, L. Vercammen, E. M. Strome, C. Konradi, and M. A. Cenci, "Spatiotemporal pattern of striatal ERK1/2 phosphorylation in a rat model of L-DOPA-induced dyskinesia and the role of dopamine D1 receptors," Biological Psychiatry, vol. 62, no. 7, pp. 800-810, 2007.

[9] M. A. Cenci, A. Tranberg, M. Andersson, and A. Hilbertson, "Changes in the regional and compartmental distribution of FosB- and JunB-like immunoreactivity induced in the dopamine-denervated rat striatum by acute or chronic L-DOPA treatment," Neuroscience, vol. 94, no. 2, pp. 515-527, 1999.

[10] J. P. Doucet, Y. Nakabeppu, P. J. Bedard et al., "Chronic alterations in dopaminergic neurotransmission produce a persistent elevation of deltaFosB-like protein(s) in both the rodent and primate striatum," European Journal of Neuroscience, vol. 8, pp. 365-381, 1996.

[11] E. Santini, E. Valjent, A. Usiello et al., "Critical involvement of cAMP/DARPP-32 and extracellular signal-regulated protein kinase signaling in L-DOPA-induced dyskinesia," The Journal of Neuroscience, vol. 27, no. 26, pp. 6995-7005, 2007.

[12] M. Lebel, L. Chagniel, G. Bureau, and M. Cyr, "Striatal inhibition of PKA prevents levodopa-induced behavioural and molecular changes in the hemiparkinsonian rat," Neurobiology of Disease, vol. 38, no. 1, pp. 59-67, 2010.

[13] X. Cao, T. Yasuda, S. Uthayathas et al., "Striatal overexpression of $\Delta$ FosB reproduces chronic levodopa-induced involuntary movements," The Journal of Neuroscience, vol. 30, no. 21, pp. 7335-7343, 2010.

[14] E. Bezard, M. P. Hill, A. R. Crossman et al., "Levetiracetam improves choreic levodopa-induced dyskinesia in the MPTPtreated macaque," European Journal of Pharmacology, vol. 485, no. 1-3, pp. 159-164, 2004.

[15] M. P. Hill, J. M. Brotchie, A. R. Crossman et al., "Levetiracetam interferes with the L-dopa priming process in MPTP-lesioned drug-naive marmosets," Clinical Neuropharmacology, vol. 27, no. 4, pp. 171-177, 2004.

[16] R. C. Boldry, S. M. Papa, A. M. Kask, and T. N. Chase, "MK-801 reverses effects of chronic levodopa on D1 and D2 dopamine agonist-induced rotational behavior," Brain Research, vol. 692, no. 1-2, pp. 259-264, 1995.

[17] Y. Xu, Z. Zhang, K. Qin, S. M. Papa, and X. Cao, "Quantitative autoradiographic study on receptor regulation in the basal ganglia in rat model of levodopa-induced motor complications," Journal of Huazhong University of Science and TechnologyMedical Science, vol. 29, no. 2, pp. 156-162, 2009.

[18] M. Lundblad, B. Picconi, H. Lindgren, and M. A. Cenci, "A model of L-DOPA-induced dyskinesia in 6-hydroxydopamine lesioned mice: relation to motor and cellular parameters of nigrostriatal function," Neurobiology of Disease, vol. 16, no. 1, pp. 110-123, 2004. 
[19] F. Stocchi, M. Tagliati, and C. W. Olanow, "Treatment of levodopa-induced motor complications," Movement Disorders, vol. 23, no. 3, pp. S599-S612, 2008.

[20] T. N. Chase and J. D. Oh, "Striatal mechanisms and pathogenesis of Parkinsonian signs and motor complications," Annals of Neurology, vol. 47, no. 4, pp. S122-S130, 2000.

[21] S. M. Papa, Y. P. Auberson, and J. T. Greenamyre, "Prolongation of levodopa responses by glycine ${ }_{B}$ antagonists in parkinsonian primates," Annals of Neurology, vol. 56, no. 5, pp. 723-727, 2004.

[22] I. Aubert, C. Guigoni, K. Håkansson et al., "Increased D1 dopamine receptor signaling in levodopa-induced dyskinesia," Annals of Neurology, vol. 57, no. 1, pp. 17-26, 2005.

[23] E. Santini, V. Sgambato-Faure, Q. Li et al., "Distinct changes in cAMP and extracellular signal-regulated protein kinase signalling in L-DOPA-induced dyskinesia," PLoS ONE, vol. 5, no. 8, Article ID e12322, 2010.

[24] A. Berthet and E. Bezard, "Dopamine receptors and 1-dopainduced dyskinesia," Parkinsonism and Related Disorders, vol. 15, no. 4, pp. S8-S12, 2009.

[25] O. Berton, C. Guigoni, Q. Li et al., "Striatal overexpression of DeltaJunD resets L-DOPA-induced dyskinesia in a primate model of Parkinson disease," Biological Psychiatry, vol. 66, no. 6, pp. 554-561, 2009.

[26] P. K. Tekumalla, F. Calon, Z. Rahman et al., "Elevated levels of $\triangle$ FosB and RGS9 in striatum in Parkinson's disease," Biological Psychiatry, vol. 50, no. 10, pp. 813-816, 2001.

[27] J. J. Cereghino, V. Biton, B. Abou-Khalil, F. Dreifuss, L. J. Gauer, and I. Leppik, "Levetiracetam for partial seizures: results of a double-blind, randomized clinical trial," Neurology, vol. 55, no. 2, pp. 236-242, 2000.

[28] C.-H. Lee, C.-Y. Lee, T.-S. Tsai, and H.-H. Liou, "PKA-mediated phosphorylation is a novel mechanism for levetiracetam, an antiepileptic drug, activating ROMK1 channels," Biochemical Pharmacology, vol. 76, no. 2, pp. 225-235, 2008.

[29] S. M. Papa, “The cannabinoid system in Parkinson's disease: multiple targets to motor effects," Experimental Neurology, vol. 211, no. 2, pp. 334-338, 2008.

[30] K. E. Lyons and R. Pahwa, "Efficacy and tolerability of levetiracetam in Parkinson disease patients with levodopa-induced dyskinesia," Clinical Neuropharmacology, vol. 29, no. 3, pp. 148153, 2006.

[31] B. Tousi and T. Subramanian, "The effect of levetiracetam on levodopa induced dyskinesia in patients with Parkinson's disease," Parkinsonism and Related Disorders, vol. 11, no. 5, pp. 333-334, 2005.

[32] T. A. Zesiewicz, K. L. Sullivan, J. L. Maldonado, W. O. Tatum, and R. A. Hauser, "Open-label pilot study of levetiracetam (Keppra) for the treatment of levodopa-induced dyskinesias in Parkinson's disease," Movement Disorders, vol. 20, no. 9, pp. 1205-1209, 2005.

[33] P. Stathis, S. Konitsiotis, G. Tagaris, and D. Peterson, "Levetiracetam for the management of levodopa-induced dyskinesias in Parkinson's disease," Movement Disorders, vol. 26, no. 2, pp. 264-270, 2011.

[34] M. A. Cenci and C. Konradi, "Maladaptive striatal plasticity in 1DOPA-induced dyskinesia," Progress in Brain Research, vol. 183, pp. 209-233, 2010. 


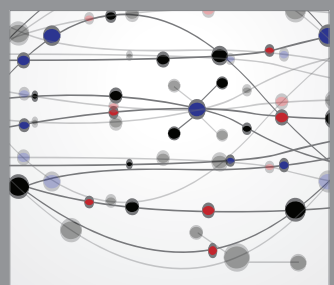

The Scientific World Journal
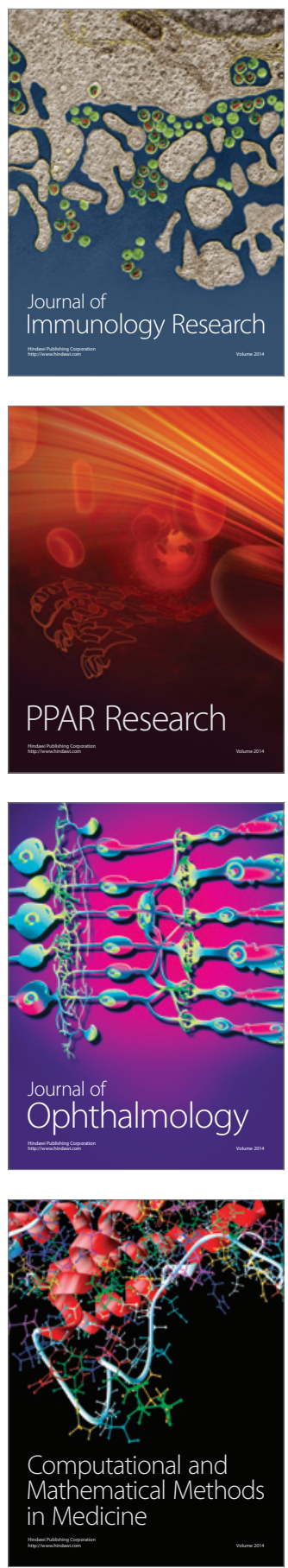

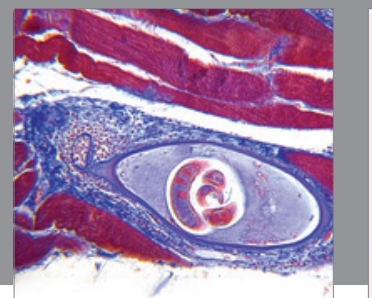

Gastroenterology

Research and Practice
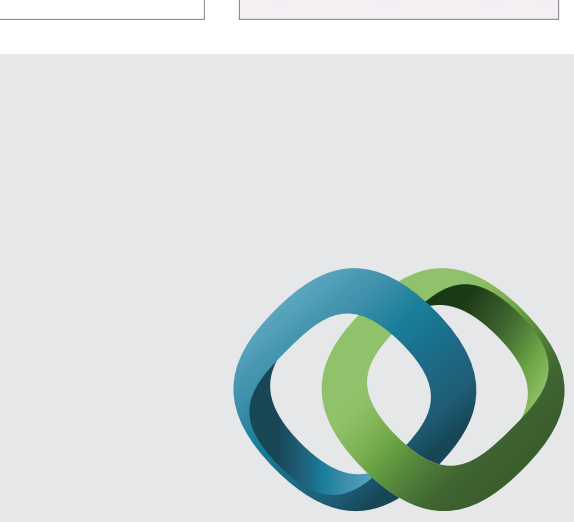

\section{Hindawi}

Submit your manuscripts at

http://www.hindawi.com
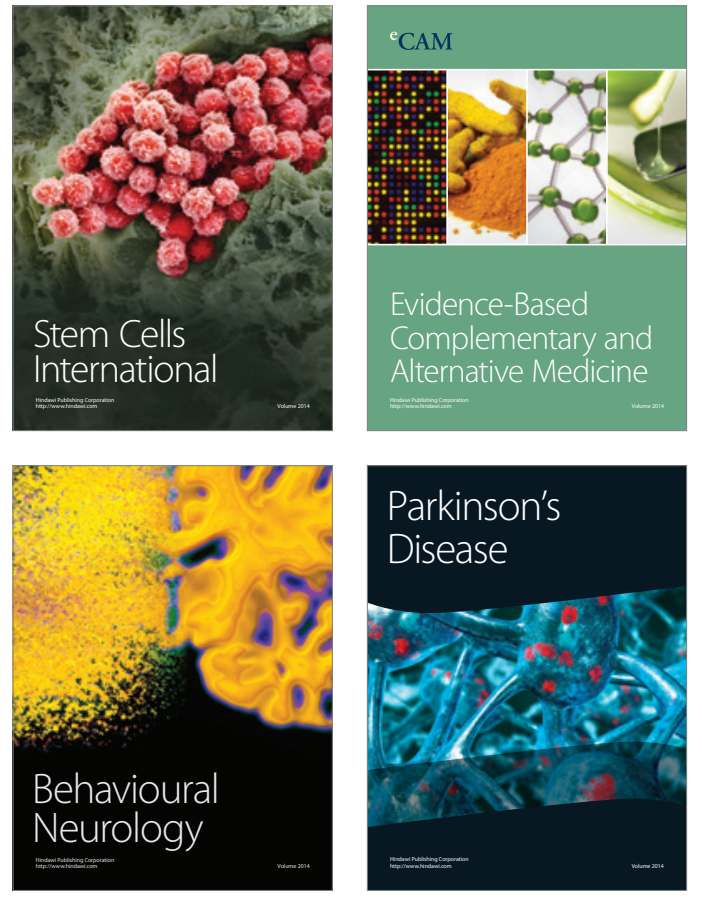
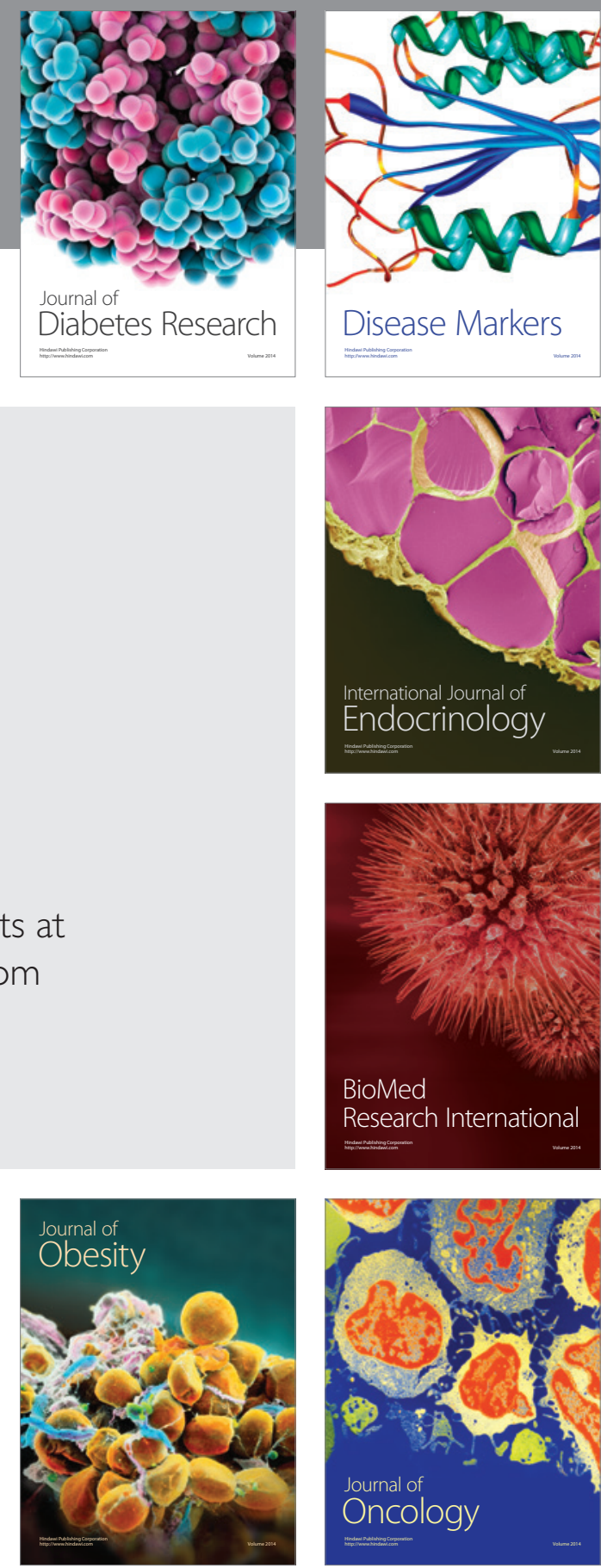

Disease Markers
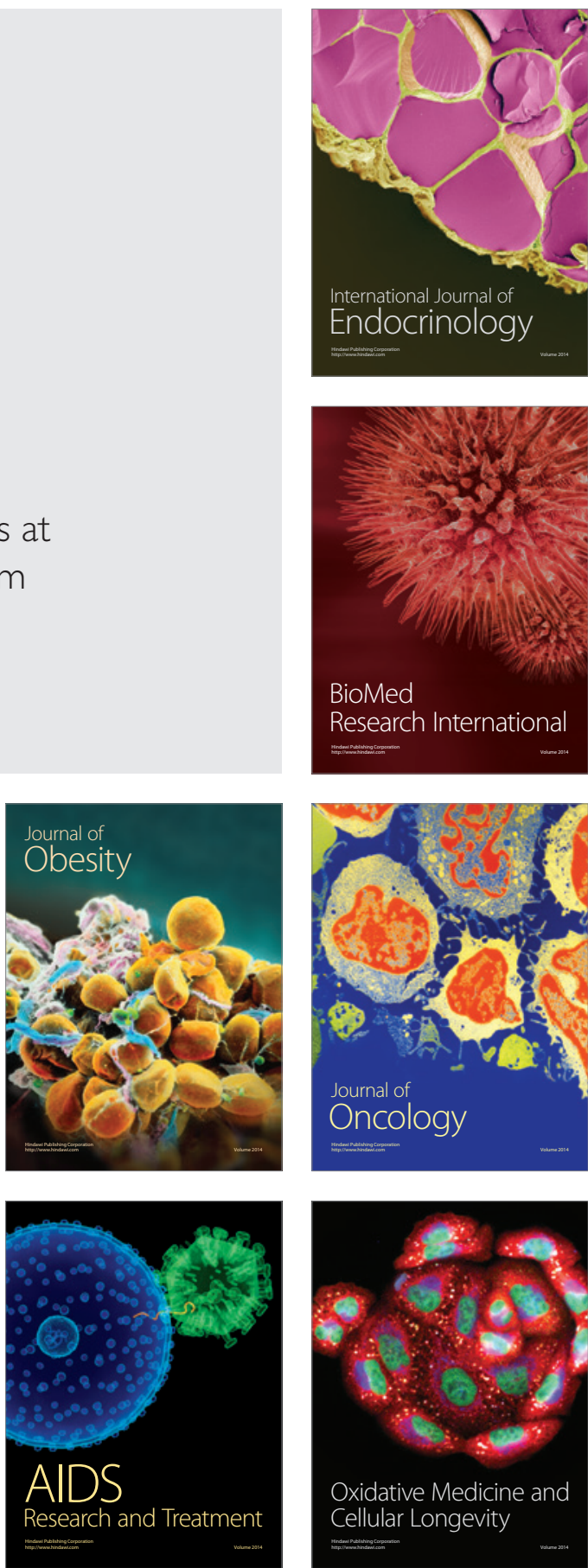